\title{
Hydrodynamics of the quantum Hall smectics
}

\author{
Michael M. Fogler \\ School of Natural Sciences, Institute for Advanced Study, Olden Lane, Princeton, New Jersey 08540
}

Valerii M. Vinokur

Material Science Division, Argonne National Laboratory, Argonne, Illinois 60439

We propose a dynamical theory of the stripe phase arising in a two-dimensional electron liquid near half-integral fillings of high Landau levels. The system is modelled as a novel type of a smectic liquid crystal with the Lorentz force dominated dynamics. We calculate the structure factor, the dispersion relation of the collective modes, and their intrinsic attenuation rate. We show that thermal fluctuations cause a strong power-law renormalization of the elastic and dissipative parameters familiar from the conventional smectics but with different dynamical scaling exponents.

Planar arrays of interacting lines or stripes have become a paradigm for many different physical systems, including domain walls in magnets, layered superconductors, biophysical systems, liquid crystals, and charge density waves (CDW) 国. A unidirectional CDW or the stripe phase was also predicted [2] to form in GaAs twodimensional (2D) electron systems when the occupation of the third or a higher Landau level is close to $\frac{1}{2}$ [3]. This prediction was attested by a recent discovery of a dramatic magnetotransport anisotropy [4] in the indicated range of filling factors. The easy (low resistance) and hard (high resistance) current directions are thought to be along and across the stripes, respectively. In order to have a better foundation for the magnetotransport studies, one has to first understand the intrinsic dynamics of the stripe phase, without intervening disorder effects [5]. Recent investigations [6 \& 8 revolved around the instability of the stripes against the $2 k_{F}$-modulation along the direction of the translational order, which would transform the system into a highly anisotropic Wigner crystal. According to MacDonald and Fisher [8], such an instability appears only at temperatures $T$ below $1 \mathrm{mK}$. Here we would like to address another interesting regime of relatively high $T$ where the $2 k_{F}$ modulation is absent and moreover, the sole periodic modulation is due to the main CDW harmonic with wave vector $q_{0}$. In classical terms, the electron density at the topmost Landau level is of the form $n(\mathbf{r}, t)+\operatorname{Re} \Psi(\mathbf{r}, t)$, where $\Psi=|\Psi| e^{i q_{0}(x-u)}$ is the CDW order parameter. (We chose the $\hat{\mathbf{x}}$-direction to be perpendicular to the stripes). Although oversimplified, this classical picture correctly identifies the low-frequency long-wavelength degrees of freedom: the coarse-grained component $n$ of the total density, and the CDW phase (or the displacement field) $u$. It is natural to expect that at sufficiently small $\omega$ and $q$ the dynamics of $u$ and $n$ is governed by a certain hydrodynamic theory. The formulation of such a theory is the subject of this Letter.

We begin by writing down an effective Hamiltonian, from which we derive the hydrodynamic equations of mo- tion, the spectrum of the collective modes, and the structure factor. Next, we study the renormalization of the bare parameters of the theory by thermal fluctuations. Our results can be verified by measuring the transmission of the micro- and surface acoustic waves as well as inelastic light scattering techniques provided the samples are of high enough mobility so that the disorder effects, which we ignore here, are not important.

Effective Hamiltonian.- Our first step is to construct an effective Hamiltonian for $n$ and $u$, which is (a) rotationally and translationally invariant and (b) reflects the specific properties of the system, such as the long-range Coulomb interaction. Very useful in this process is the similarity [6] to smectic liquid crystals [9]. The resultant form of the effective Hamiltonian is as follows:

$$
\begin{array}{r}
H=\frac{1}{2} \int d^{2} r\left[Y E^{2}(u)+E(u) \Delta+K\left(\nabla^{2} u\right)^{2}\right. \\
\left.+\chi^{-1} \delta n^{2}+\Phi n+2 C E(u) \delta \rho+\rho v^{2}\right],
\end{array}
$$

where $E(u)=\nabla_{x} u-\frac{1}{2}(\nabla u)^{2}$ in the usual rotationally invariant strain [9], $Y$ and $K$ are the compression and bending elastic moduli, $\chi$ is the compressibility [10], $\Delta$ is an auxillary parameter (counter-term), needed to guarantee the condition $\left\langle\nabla_{x} u\right\rangle=0, \rho=m n$ is the mass density, $\delta \rho=\rho-\rho_{0}$ is its deviation from the equilibrium value $\rho_{0}=m n_{0} . \Phi(\mathbf{r})=\int d^{2} r^{\prime} \delta n\left(\mathbf{r}^{\prime}\right) U\left(\mathbf{r}-\mathbf{r}^{\prime}\right)$, is the electrostatic (Hartree) potential, with $U(r)=e^{2} / \kappa r$ at large $r$ (see more details in [2]). The penultimate term in Eq. (Ii) accounts for the dependence of the CDW periodicity on $n_{0}$, with $C=(Y / m) \partial \ln q_{0} / \partial n_{0}$. Finally, the last term is the correction to the kinetic energy, with $v$ being the velocity of the electron fluid. This term is mainly a bookkeeping device: it vanishes after the projection on a single (topmost) Landau level but enables us to derive the equations of motion via the standard Poisson-bracket method 11,12].

Equations of motion. - The hydrodynamic fluctuations of our system are governed by the equations 


$$
\begin{aligned}
F_{u} & \equiv \rho_{0} \frac{\dot{W}+(\mathbf{v} \nabla) W}{|\nabla W|}-\lambda_{p}|\nabla W| \frac{\delta H}{\delta u}=\zeta_{u}, \\
F_{j} & \equiv \frac{\partial\left(\rho v_{j}\right)}{\partial t}+\nabla_{j}\left(\rho v_{k} v_{j}\right)-\rho \omega_{c} \varepsilon_{j k} v_{k}+\nabla_{j} W \frac{\delta H}{\delta u} \\
& +L_{j k}(i \nabla) v_{k}+\rho \nabla_{k}\left(\chi^{-1} \delta \rho+\Phi+C E\right)=\zeta_{j},
\end{aligned}
$$

and the continuity equation $\dot{\rho}+\boldsymbol{\nabla}(\rho \mathbf{v})=0$. The notations here are as follows: $W \equiv x-u, \varepsilon_{j k}$ is the unit antisymmetric tensor, and $\omega_{c}=e B / m c$ is the cyclotron frequency. Functions $L_{j k}(\mathbf{q})$ describe dissipation. As in $3 \mathrm{D}$ case [9], they can be parametrized by viscosities $\eta_{i}$,

$$
\begin{aligned}
L_{j k}(\mathbf{q}) & =\eta_{4} q_{j} q_{k}+\delta_{j k} \eta_{3} q_{x}^{2} \\
& +\delta_{j x} \delta_{k x}\left[\eta_{3} q^{2}+\left(\eta_{1}-4 \eta_{3}+\eta_{4}-2 \eta_{5}\right) q_{x}^{2}\right] \\
& +\left(\eta_{3}-\eta_{4}+\eta_{5}\right) q_{x}\left(q_{j} \delta_{k x}+q_{k} \delta_{j x}\right) .
\end{aligned}
$$

(There is no analog of $\eta_{2}$ in 2D). One more dissipative coefficient, $\lambda_{p}$, [Eq. (2)] describes the permeation, i.e., the mass transport across the stripes. At relatively high $T$, such that $|\Psi| \lesssim n_{0}, \lambda_{p} \sim\left(\eta_{3} q_{0}^{2} / \rho_{0} \omega_{c}^{2}\right)\left(n_{0} /|\Psi|\right)^{2}$. Finally, $\zeta_{a}$ 's in Eqs. (2) and (3) are the Langevin noises, which satisfy the fluctuation-dissipation theorem,

$$
\begin{aligned}
& \left\langle\zeta_{u}(1) \zeta_{k}(2)\right\rangle=0, \quad 1(2) \equiv\left\{\mathbf{r}_{1(2)}, t_{1(2)}\right\}, \\
& \left\langle\zeta_{u}(1) \zeta_{u}(2)\right\rangle=2 k_{B} T \lambda_{p} \rho_{0} \delta(1-2), \\
& \left\langle\zeta_{j}(1) \zeta_{k}(2)\right\rangle=2 k_{B} T L_{j k}(i \boldsymbol{\nabla}) \delta(1-2) .
\end{aligned}
$$

The main difference of our model from the conventional smectics is the presence of the strong magnetic field. As we will see below, it drastically changes both the linearized and nonlinear dynamics of the system.

Field-theory description.- Our ultimate goal is to calculate various correlation functions, e.g., the dynamical structure factor,

$$
S(\mathbf{q}, \omega)=\left(N_{0} m^{2}\right)^{-1} \int d t e^{i \omega t}\left\langle\rho_{\mathbf{q}}(t) \rho_{-\mathbf{q}}(0)\right\rangle,
$$

where $N_{0}$ is the total number of electrons at the topmost Landau level, and $\langle\ldots\rangle$ stands for the thermal averaging, or equivalently, the averaging over the Langevin noise. The latter can be done in a systematic way by means of the Martin-Siggia-Rose (MSR) formalism [13], whose field-theoretic version was previously applied to the conventional smectics by Kats and Lebedev [12].

The implementation of the MSR method begins with enforcing the equations of motion by $\delta$-function-type weight factors, $\delta\left(F_{a}-\zeta_{a}\right)$, in the path integral over $F_{a}$. These $\delta$-functions are then represented by integrals of $\exp \left[i p_{a}\left(F_{a}-\zeta_{a}\right)\right]$ over auxillary dynamical variables $p_{a}$, which enables one to average over the Gaussian noise $\zeta_{a}$. Finally, one changes the integration variables from $F_{a}$ to $V_{i} \equiv \rho v_{i} / \rho_{0}$ and $u$. If the denote by $F_{a}^{n l}$ the nonliner terms in $F_{a}$, and introduce vectors $\phi^{\dagger}=\left\{V^{\dagger}, P^{\dagger}\right\}$, $V^{\dagger}=\left\{u, V_{x}, V_{y}\right\}, P^{\dagger}=\left\{p_{u}, p_{x}, p_{y}\right\}$, then the resultant action becomes

$$
\begin{aligned}
& A=-i \int d t \int d^{2} r p_{a} F_{a}^{n l}(u, v)-\ln J+A_{0}, \\
& A_{0}=\frac{1}{2} \int \frac{d^{2} q}{(2 \pi)^{2}} \int \frac{d \omega}{2 \pi} \boldsymbol{\phi}_{\mathbf{q} \omega}^{\dagger} \mathbf{G}_{0}^{-1}(\mathbf{q}, \omega) \boldsymbol{\phi}_{\mathbf{q} \omega}
\end{aligned}
$$

where $J=\left|\operatorname{det} \partial F_{a} / \partial V_{b}\right|$ is the Jacobian and $\mathbf{G}_{0}$ is the (bare) propagator of the following block-matrix form

$$
\mathbf{G}_{0}=\left[\begin{array}{cc}
\mathbf{G}_{V V} & -i \mathbf{G}_{V P} \\
-i \mathbf{G}_{V P}^{\dagger} & \mathbf{0}
\end{array}\right] .
$$

Two particular components of $\mathbf{G}_{0}, G_{u u}$ and $G_{u p_{x}}$,

$$
\begin{aligned}
G_{u p_{x}}(\mathbf{q}, \omega) & =\frac{-i s^{2}}{Q(\mathbf{q}) s^{2}-i \omega \tilde{\nu}(\mathbf{q})-\omega^{2} \frac{\omega_{c}^{2}}{\omega_{p}^{2}(q)}}, \\
G_{u u}(\mathbf{q}, \omega) & =\frac{k_{B} T}{\rho_{0} \omega}\left[G_{u p_{x}}(\mathbf{q}, \omega)-G_{u p_{x}}(\mathbf{q},-\omega)\right],
\end{aligned}
$$

will play an important role in the later discussion. Here we introduced the notations $Q(\mathbf{q})=\left(Y q_{x}^{2}+K q^{4}\right) / \rho_{0}$, $\omega_{p}^{2}(q)=n_{0} q^{2}\left[U(q)+\chi^{-1}\right] / m, \tilde{\nu}_{i}=\eta_{i} q^{2} / \rho_{0}, c=q_{x} / q=$ $\cos \theta, s=q_{y} / q=\sin \theta$, and

$$
\tilde{\nu}(\mathbf{q})=\tilde{\nu}_{3}+c^{2} s^{2}\left(\tilde{\nu}_{1}-4 \tilde{\nu}_{3}+\tilde{\nu}_{4}-2 \tilde{\nu}_{5}\right) .
$$

Equations (12) and (13) are obtained by neglecting $\omega, \lambda_{p}$, $\tilde{\nu}_{i}$, and $C$ compared to quantities proportional to "large" frequencies $\omega_{c}$ and $\omega_{p}$.

Harmonic theory.- If only the quadratic part $A_{0}$ of the full action is retained, then the correlators of the velocity fields $V_{a}$ are given by the components $G_{V_{a} V_{b}}$ of $\mathbf{G}_{0}$ and can be found after straightforward albeit tedious algebra. The continuity equation enables us to relate these correlators to the structure factor. This way we get

$S(\mathbf{q}, \omega)=\frac{2}{m \omega} \operatorname{Im} \frac{k_{B} T q^{2}\left(Q s^{2}-i \omega \tilde{\nu}\right)}{Q \omega_{p}^{2}\left(s^{2}+\lambda_{p} \tilde{\nu}\right)-i \omega \omega_{c}^{2} \alpha-\omega^{2} \omega_{c}^{2}}$,

for $\omega \ll \omega_{c}$ and, in particular,

$$
S(\mathbf{q}, 0)=2 k_{B} T \frac{q^{2}}{m \omega_{p}^{4}} \frac{\omega_{c}^{2} \lambda_{p}+c^{2} \tilde{\nu}_{3}+s^{2} \tilde{\nu}_{4}}{s^{2}+\lambda_{p} \tilde{\nu}} .
$$

The poles of $S(\mathbf{q}, \omega)$ correspond to the collective modes. Two of them (magnetophonons) are gapless:

$$
\omega_{m}(\mathbf{q}) \simeq \sin 2 \theta\left(\frac{Y}{\rho_{0}}\right)^{1 / 2} \frac{\omega_{p}(q) q}{2 \omega_{c}}-\frac{i}{2} \alpha(\mathbf{q})
$$

and its counterpart $\omega=-\omega_{m}^{*}(\mathbf{q})$. Their attenuation rate $\alpha(\mathbf{q})$ is given by

$$
\alpha(\mathbf{q})=\lambda_{p} Q+\omega_{c}^{-2}\left[\omega_{p}^{2}(q) \tilde{\nu}+Q\left(\tilde{\nu}_{3} c^{2}+\tilde{\nu}_{4} s^{2}\right)\right],
$$

up to terms proportional to $C$ and higher powers of $\tilde{\nu}_{i}$ and $\lambda_{p}$. The other two collective modes (magnetoplasmons) 
have a large gap $\omega_{c}$. Thus, the number of the hydrodynamic (gapless) modes coincides with the number of the hydrodynamic variables $(n$ and $u$ ) as it should [14].

For Coulomb interaction $\omega_{p}(q) \propto q^{1 / 2}$; hence, within the harmonic theory the magnetophonons have $\omega_{m}(\mathbf{q}) \propto$ $q^{3 / 2}$ dispersion, similar to that of the Wigner crystal 15] but with the $\theta$-dependent prefactor. Propagating magnetophonon modes exist as long as $\theta$ is not too small so that $\operatorname{Re} \omega_{m}(\mathbf{q}) \gg \alpha(\mathbf{q})$ in Eq. (17); otherwise, they are replaced by the two overdamped modes:

$\omega_{\text {fast }}(\mathbf{q}) \simeq-i \alpha(\mathbf{q}), \quad \omega_{\text {slow }}(\mathbf{q}) \simeq-\frac{i}{\alpha(\mathbf{q})}\left(\frac{q_{x} q_{y}}{q} \frac{\omega_{p}}{\omega_{c}}\right)^{2} \frac{Y}{\rho_{0}}$.

Below we will see that at small enough $q$ the magnetophonon dispersion relation and their damping are significantly modified by the anharmonisms.

Renormalization. Our next step is to calculate the propagator $\mathbf{G}=\left(\mathbf{G}_{0}^{-1}+\boldsymbol{\Sigma}\right)^{-1}$ of the full theory, Eq. (9), treating the previously ignored nonlinear terms as perturbations. We expect that $\mathbf{G}$ has the same form as $\mathbf{G}_{0}$ but with $Y, K$, and other parameters replaced by $\omega$ and $q$-dependent (renormalized) values $Y^{R}(\mathbf{q}, \omega)$, $K^{R}(\mathbf{q}, \omega)$, etc. The perturbative corrections to $Y, K$, and $C$ are determined by the self-energy component $\Sigma_{u p_{x}}(\mathbf{q}, \omega)$. Similarly, the corrections to $\nu_{i}$ 's are determined by $\Sigma_{p_{j} p_{k}}(\mathbf{q}, \omega)$. To the lowest order in $T$ they are given by the diagrams shown in Fig. 1 .

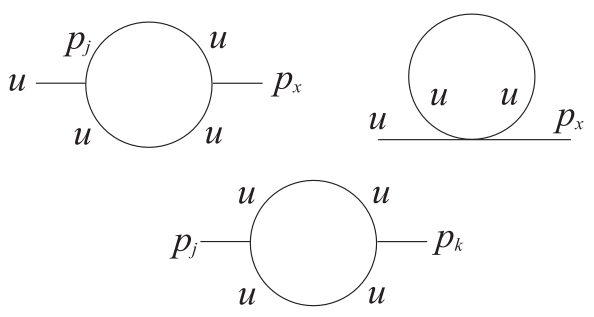

FIG. 1. Self-energy diagrams.

Doing the power counting with the help of Eqs. (12), (13), and the relation $k_{x} \sim k^{2}(K / Y)^{1 / 2}$, where $k$ is the loop momentum, we quickly discover that $\Sigma_{u p_{x}}$ and $\Sigma_{p_{j} p_{k}}$ are infrared-divergent. To resolve this problem, we utilize the renormalization group (RG) procedure formulated in $d=3-\epsilon$ spatial dimensions [one $x$-dimension plus $(d-1)$ " $\perp$ "-dimensions]. Each step of our RG transformation consists of integrating out the cylindrical shell $\Lambda e^{-l}<k_{\perp}<\Lambda$ in the loop diagrams of Fig. 1, followed by rescaling of the momenta $k=k^{\prime} e^{-l}$ to restore the ultraviolet cutoff $\Lambda \sim q_{0}$ on $k_{\perp}$. It is convenient to rescale the $u$-field as well $u(\mathbf{r})=u^{\prime}\left(\mathbf{r}^{\prime}\right) e^{l}$ to preserve the structure of $E(u)$. We did not find it necessary to rescale the frequencies or impose a cutoff on $k_{x}$ [16]. The one-loop RG equations are given by

$$
\begin{aligned}
\frac{d}{d l} Y= & d Y-\frac{g}{8} Y, \quad \frac{d}{d l} C=d C-\frac{g}{8} C, \\
\frac{d}{d l} K= & (d-2) K+\frac{g}{8(d-1)} K, \\
\frac{d}{d l} g= & (3-d) g-\frac{d+2}{16(d-1)} g^{2}, \\
\frac{d}{d l} \nu_{3}^{2}= & 2 d \nu_{3}^{2}+\frac{g}{2(d-1)} \\
\times & {\left[\frac{\omega_{c}^{2}}{\omega_{p}^{2}\left(\Lambda e^{-l}\right)} \frac{K(l)}{\rho_{0}} e^{(d+2) l}+\frac{\nu_{3}^{2}}{4}\right], }
\end{aligned}
$$

where $g \equiv k_{B} T Y^{1 / 2} K^{-3 / 2} \Lambda^{d-3} / S_{d-1}$ is the dimensionless coupling constant, with $S_{d-1}=(4 \pi)^{\frac{d-1}{2}} \Gamma\left(\frac{d-1}{2}\right) / 2$. Equations for other viscosities are similar to (22) and are not shown.

The solutions of the RG equations (19 22) are as follows:

$$
\begin{aligned}
& g(l)=g_{*}+\frac{g_{0}-g_{*}}{D}, \quad D \equiv 1+\frac{g_{0}}{g_{*}}\left[e^{(3-d) l}-1\right], \quad \nu_{3}^{2}(l)=e^{2 d l}\left[\nu_{3}^{2}(0)+\frac{1}{2(d-1)} \frac{\omega_{c}^{2}}{\omega_{p}^{2}\left(\Lambda e^{-l}\right)} \frac{K_{0}}{\rho_{0}}\right] D^{\frac{2}{(d+2)}}, \\
& Y(l)=Y_{0} e^{d l} D^{-\gamma_{Y}}, \quad C(l)=C_{0} e^{d l} D^{-\gamma_{Y}}, \quad \gamma_{Y}=2 \frac{d-1}{d+2}, \quad K(l)=K_{0} e^{(d-2) l} D^{\gamma_{K}}, \quad \gamma_{K}=\frac{2}{d+2} .
\end{aligned}
$$

As one can see, for small $\epsilon=3-d, g$ flows to a weak coupling fixed point $g_{*}=16(3-d)(d-1) /(d+2)$, justifying our one-loop RG. The renormalized values of the parameters of the harmonic theory are found by rescaling back to the original coordinates, $Y^{R}(\omega, \mathbf{q})=Y(l) e^{-d l}$, $K^{R}(\omega, \mathbf{q})=K(l) e^{-(d-2) l}, \nu_{3}^{R}(\omega, \mathbf{q})=\nu_{3}(l) e^{-d l}$, etc., where $l$ is the smallest of the three cutoffs $l_{x}, l_{\perp}, l_{\omega}$ to be found from equations $K\left(l_{x}\right) \Lambda^{4}=q_{x}^{2} e^{2 l_{x}} Y\left(l_{x}\right), \Lambda=$ $q_{\perp} e^{l_{\perp}}$, and $\omega_{c}^{2} \omega=\omega_{p}^{2}\left(\Lambda e^{-l_{\omega}}\right) \nu_{3}\left(l_{\omega}\right) \Lambda^{2} e^{-(d+2) l_{\omega}}$. These equations give rise to the characteristic crossover length and frequency scales $\xi_{x}=\left(K_{0}^{5} / Y_{0}\right)^{1 / 2} /\left(k_{B} T\right)^{2}, \xi_{y}=$ $\left(K_{0}^{3} / Y_{0}\right)^{1 / 2} / k_{B} T$, and $\omega_{y}=\left(K_{0} / \rho_{0}\right)^{1 / 2} \omega_{p}\left(\xi_{y}^{-1}\right) / \omega_{c} \xi_{y}^{2}$.

Taking Eq. (24) for the face value, we obtain $\gamma_{Y}=$ $\gamma_{K}=\frac{1}{2}$ in $2 \mathrm{D}(\epsilon=1)$ and discover the following three types of asymptotic behavior:

$$
\begin{aligned}
Y^{R} & \sim Y_{0}\left(\xi_{y} q_{y}\right)^{1 / 2}, \quad K^{R} \sim K_{0}\left(\xi_{y} q_{y}\right)^{-1 / 2}, \\
\nu_{3}^{R} & \sim \frac{K_{0}}{\rho_{0} \omega_{y} \xi_{y}^{2}\left(q_{y} \xi_{y}\right)^{3 / 4}}, \quad \nu_{i}^{R} \sim \frac{a_{i} Y_{0}}{\rho_{0} \omega_{y}\left(q_{y} \xi_{y}\right)^{7 / 4}}
\end{aligned}
$$




$$
\begin{aligned}
\text { for } q_{x} & \ll \xi_{x}^{-1}\left(q_{y} \xi_{y}\right)^{3 / 2}, q_{y} \ll \xi_{y}^{-1}, \omega \ll \omega_{y}\left(q_{y} \xi_{y}\right)^{9 / 4}, \\
Y^{R} & \sim Y_{0}\left(\xi_{x} q_{x}\right)^{1 / 3}, \quad K^{R} \sim K_{0}\left(\xi_{x} q_{x}\right)^{-1 / 3}, \\
\nu_{3}^{R} & \sim \frac{K_{0}}{\rho_{0} \omega_{y} \xi_{y}^{2}\left(q_{x} \xi_{x}\right)^{1 / 2}}, \quad \nu_{i}^{R} \sim \frac{a_{i} Y_{0}}{\rho_{0} \omega_{y}\left(q_{x} \xi_{x}\right)^{7 / 6}}
\end{aligned}
$$

for $q_{x} \ll \xi_{x}^{-1}, q_{y} \ll \xi_{y}^{-1}\left(q_{x} \xi_{x}\right)^{2 / 3}, \omega \ll \omega_{y}\left(q_{x} \xi_{x}\right)^{3 / 2}$, and

$$
\nu_{3}^{R} \sim \frac{K_{0}}{\rho_{0} \xi_{y}^{2}} \omega_{y}^{-2 / 3} \omega^{-1 / 3}, \quad \nu_{i}^{R} \sim \frac{a_{i} Y_{0}}{\rho_{0} \omega_{y}^{2 / 9} \omega^{7 / 9}}
$$

for $\omega \gg \omega_{y}\left(q_{y} \xi_{y}\right)^{9 / 4}, \omega \gg \omega_{y}\left(q_{x} \xi_{x}\right)^{3 / 2}$. Here $a_{1}=$ $(1-r)^{2}, a_{4}=r^{2}, a_{5}=(1-r) r$, and $r=\rho_{0} C_{0} / Y_{0}$. Thus, $a_{1} a_{4}=a_{5}^{2}$, which entails the relation [11] $\Delta \eta_{1} \Delta \eta_{4}=$ $\left(\Delta \eta_{5}\right)^{2}$ derived earlier for the conventional smectics.

Discussion. Our one-loop-level results for $Y^{R}$ and $K^{R}$ turn out to be exact [17] for the static limit [Eqs. (25) and (26)]. It is possible that this success transcends to the dynamics, in which case the scaling exponents for viscosities in Eqs. (25) 27) would be exact as well.

Within the domain of anomalous hydrodynamics summarized by Eqs. (25 27), the magnetophonon dispersion relation becomes

$$
\begin{aligned}
& \operatorname{Re} \omega_{m}(\mathbf{q}) \sim s c^{7 / 6}\left(\xi_{x} q\right)^{5 / 3} \frac{\omega_{p}\left(\xi_{x}^{-1}\right)}{\omega_{c} \xi_{x}} \sqrt{\frac{Y_{0}}{\rho_{0}}} \\
& \operatorname{Im} \omega_{m}(\mathbf{q}) \sim s^{2} c^{5 / 6}\left(\xi_{x} q\right)^{11 / 6}(1-2 r)^{2} \frac{\omega_{y}}{\xi_{y}} \sqrt{\frac{K_{0}}{Y_{0}}}
\end{aligned}
$$

for a finite fixed $\theta$ and $q \ll \xi_{x}^{-1}$. In contrast to the case of conventional smectics [11.12], the scaling of $\operatorname{Im} \omega_{m}(\mathbf{q})$ is cut off by $l_{x}$ [Eq. (26)] not $l_{\omega}$ [Eq. (27)].

Strictly speaking, the smectic behavior in $2 \mathrm{D}$ can persist only up to the length scale smaller than the average distance $\xi_{d} \sim \exp \left(E_{d} / 2 k_{B} T\right)$ between dislocations, $E_{d} \sim K_{0}$ being the dislocation energy [18]. The anomalous hydrodynamics can be observed if $\xi_{x}<\xi_{d}$, which is satisfied at low $T$ where $\xi_{d}$ is exponentially large [19].

In conclusion, we formulated a novel long-wavelength low-frequency effective theory of the stripe phase arising in a two-dimensional electron liquid near half-integral fillings of high Landau levels. Our theory applies at relatively high temperatures and in the clean limit. We demonstrated that the collective mode properties of the system exhibit nontrivial power-law scaling, which can be verified by microwave or surface acoustic wave measurements at a finite wave vector or by inelastic light scattering experiments. Our future plans include applying the proposed hydrodynamic approach to the problem of the magnetotransport.

Acknowledgements.- One of us (M. F.) is grateful to B. I. Shklovskii for stimulating his interest to hydrodynamic problems and also to S. Simon and L. Pryadko for comments on the manuscript. This work is supported by the U.S. Department of Energy under grants Nos. DEFG02-90ER40542 and W-31-109-ENG-38, and also by the NSF under grant No. DMR-91-20000.

[1] M. Seul and D. Andelman, Science, 267, 476 (1995).

[2] A. A. Koulakov, M. M. Fogler, and B. I. Shklovskii, Phys. Rev. Lett. 76, 499 (1996); Phys. Rev. B 54, 1853 (1996); R. Moessner and J. T. Chalker, ibid. 54, 5006 (1996).

[3] M. M. Fogler and A. A. Koulakov, Phys. Rev. B 55, 9326 (1997); E. H. Rezayi, F. D. M. Haldane, and K. Yang, Phys. Rev. Lett. 83, 1219 (1999) and cond-mat/0001394.

[4] M. P. Lilly, K. B. Cooper, J. P. Eisenstein, L. N. Pfeiffer, and K. W. West, Phys. Rev. Lett. 82, 394 (1999); R. R. Du, D. C. Tsui, H. L. Störmer, L. N. Pfeiffer, and K. W. West, Solid State Commun. 109, 389 (1999); M. Shayegan, H. C. Manoharan, S. J. Papadakis, and E. P. de Poortere, cond-mat/9903405.

[5] For recent theoretical work on transport, see Ref. 8]; E. Fradkin, S. A. Kivelson, E. Manousakis, and K. Nho, Phys. Rev. Lett. 84, 1982 (2000); F. von Oppen, B. I. Halperin, and A. Stern, cond-mat/9910132.

[6] E. Fradkin and S. A. Kivelson, Phys. Rev. B 59, 8065 (1999).

[7] H. A. Fertig, Phys. Rev. Lett. 82, 3693 (1999).

[8] A. H. MacDonald and M. P. A. Fisher, Phys. Rev. B 61, 5724 (2000).

[9] P. G. de Gennes and J. Prost, The Physics of Liquid Crystals (Oxford Univ. Press, New York, 1995).

[10] The parameters $Y, K$, and $\chi$ of the effective Hamiltonian are determined by the short-range physics, which involves the competition between Hartree, exchange, correlation, and the entropy parts of the free energy and can be estimated following Ref. [2].

[11] G. F. Mazenko, S. Ramaswamy, and J. Toner, Phys. Rev. Lett. 49, 51 (1982); Phys. Rev. A 28, 1618 (1983).

[12] E. I. Kats and V. V. Lebedev, Fluctuational Effects in The Dynamics of Liquid Crystals (Springer-Verlag, New York, 1994).

[13] P. C. Martin, E. D. Siggia, H. A. Rose, Phys. Rev. A 8, 423 (1973); C. De Dominicis and L. Peliti, Phys. Rev. B 18, 353 (1978).

[14] The third and last hydrodynamic variable is the energy density, which decouples from $n$ and $u$ in the approximation we are using. In a more complete theory it gives rise to the thermal diffusion (Rayleigh) mode, in addition to the four modes discussed above. Note that in the presence of the magnetic field, velocity $\mathbf{v}$ ceases to be a hydrodynamic variable.

[15] L. Bonsall and A. A. Maradudin, Phys. Rev. B 15, 1959 (1977).

[16] Similar procedure was used for a different problem by L. Radzihovsky and J. Toner, Phys. Rev. B 60, 206 (1999).

[17] L. Golubović and Z.-G. Wang, Phys. Rev. Lett. 69, 2535 (1992).

[18] J. Toner and D. R. Nelson, Phys. Rev. B 23, 316 (1981).

[19] Unless dislocations are generated quantum-mechanically even at $T=0$, leading to a "quantum nematic" phase [6]. 